\title{
Desempeño y calidad de carne de bovinos en confinamiento alimentados con diferentes niveles de subproductos agrícolas
}

\author{
Edith González-Salazar ${ }^{凶(\mathbb{0})}$ Zoot; Jesús Hemberg Duarte-Vargas ${ }^{1} \bowtie(\mathbb{0}$ Ph.D; \\ Vicente Díaz-Avila ${ }^{凶(\mathbb{0}}$ Ph.D; Román David Castañeda-Serrano ${ }^{1 *} \bowtie(\mathbb{D}$ Ph.D.
}

\begin{abstract}
${ }^{1}$ Universidad del Tolima, Facultad de Medicina Veterinaria y Zootecnia, Zootecnista, Ibagué, Colombia. ¿Universidade Estadual de Maringá, Programa de pos-graduação em Zootecnia, Maringá, Brasil.

*Correspondencia: rcastaneda@ut.edu.co
\end{abstract}

Recibido: Abril 2020; Aceptado: Febrero 2021; Publicado: Mayo 2021.

\section{RESUMEN}

Objetivo. Evaluar los efectos de diferentes niveles de concentrado fabricado con subproductos agrícolas del departamento del Huila - Colombia sobre el rendimiento y calidad de carne en bovinos confinados. Material y métodos. Se utilizaron 36 toretes F1 Bos taurus x Bos indicus con $347 \pm 20$ $\mathrm{kg}$ de peso corporal y 18 meses de edad confinados bajo condiciones de bosque seco tropical. Los tratamientos experimentales fueron niveles crecientes de concentrado elaborado con subproductos agrícolas en sustitución de pasto Pennisetum spp a razón de $85 \%$ forraje: $15 \%$ concentrado (T1); $75 \%$ forraje: $25 \%$ concentrado (T2); $65 \%$ forraje: $35 \%$ concentrado (T3) y $55 \%$ forraje: $45 \%$ concentrado (T4). Se utilizó un diseño experimental completamente al azar para evaluar las eventuales diferencias entre variables evaluadas: ganancia de peso vivo (GPV), consumo de materia seca (CMS), conversión alimenticia (CA), peso de la canal caliente (PCC), rendimiento en canal (RC), metabolitos sanguíneos y perfil de ácidos grasos en la carne. Resultados. La GPV y el peso final aumentaron con un mayor nivel de concentrado en la dieta $(p<0.05)$. No hubo diferencias en los metabolitos sanguíneos. Los ácidos grasos saturados caproico, caprílico y tridecanoico fueron menores cuando el nivel de concentrado en la dieta aumentó $(\mathrm{p}<0.05)$ mientras que, en los ácidos grasos insaturados y poliinsaturados no hubo diferencias entre los tratamientos. Conclusiones. La inclusión de subproductos agrícolas usados en el presente estudio mejora el rendimiento, el consumo de materia seca y reduce el contenido de tres ácidos grasos saturados de la carne en toretes F1 Bos taurus X Bos indicus en confinamiento bajo condiciones de bosque seco tropical.

Palabras clave: Bosque seco tropical; características de la canal; ganadería tropical; suplementación (Fuente: $C A B$ ).

\section{ABSTRACT}

Objective. Evaluate the effects of different levels of agricultural by-products concentrate on animal performance and meat quality in feedlot cattle. Material and methods. Thirty-Six F1 Bos taurus $x$ Bos indicus bulls were used with $347 \pm 20 \mathrm{~kg}$ of body weight and 18 months average of age on feedlot under tropical dry forest conditions. Experimental treatments were levels of agricultural by-

Como citar (Vancouver).

González-Salazar E, Duarte-Vargas JH, Díaz-Avila V, Castañeda-Serrano RD. Desempeño y calidad de carne de bovinos en confinamiento alimentados con diferentes niveles de subproductos agrícolas. Rev MVZ Córdoba. 2021; 26(2):e1950. https://doi.org/10.21897/rmvz.1950 
products replacing Pennisetum sp grass to $85 \%$ forage: $15 \%$ concentrate (T1); $75 \%$ forage: $25 \%$ concentrate (T2); $65 \%$ forage: $35 \%$ concentrated (T3) and 55\% forage:45\% concentrate (T4). A completely randomized experimental design was used for evaluation variables as live weight gain (LWG), dry matter intake (DMI), feed efficiency (FE), hot carcass weight (HCW), carcass yield (CY), blood metabolites and fatty acid profile in meat. Results. LWG and final weight increased with a higher level of concentrate in the diet $(p<0.043)$. There were not differences in blood metabolites. Differences in caproic, caprylic and tridecanoic saturated fatty acids were observed when level of concentrate in diet increased $(p<0.05)$ while, in unsaturated and polyunsaturated fatty acids has been not differences between treatments. Conclusions. Inclusion of agricultural by-products improved animal performance and three saturated fatty acid decreased in F1 Bos taurus $X$ Bos indicus bulls under feedlot in tropical dry forest conditions.

Keywords: Carcass characteristics; supplementation; tropical livestock; tropical dry forest (Source: $C A B$ ).

\section{INTRODUCCIÓN}

En los últimos años, la producción de carne en todo el mundo ha sido cuestionada por el uso excesivo de recursos, la generación de gases de efecto invernadero y su asociación con la presencia de residuos como hormonas y antibióticos en los productos cárnicos (1). Por lo tanto, es necesario buscar alternativas que ayuden a obtener ciclos de producción rápidos, limpios y sustentables. Con el fin de obtener una ocupación menor por animal y maximizar el rendimiento, se han utilizado sistemas de terminación en confinamiento (feedlots) principalmente en los Estados Unidos y Europa (2). La adopción de los sistemas de confinamiento, ofrecen un mejor manejo de los animales, mayor control administrativo del sistema y una alimentación controlada. Dicha maximización de los sistemas permite una mayor rentabilidad para el productor, ya que es posible establecer un alimento basado en la respuesta del animal, evitando el desperdicio y optimizando el uso del alimento ofrecido (3).

En la mayoría de los sistemas que se termina bovinos de carne en confinamiento mejora el rendimiento y la terneza de la carne, sin embargo, la principal limitante de estos sistemas es la dependencia de los costos de alimentación que incluye factores como disponibilidad, calidad y costo de materias primas, así como la relación adecuada de forraje: concentrado en la dieta (4). Por lo tanto, es necesario buscar materias primas o subproductos de bajo costo de acuerdo con la disponibilidad de materias primas en cada región o país para viabilizar estos sistemas de confinamiento e incluirlos en la proporción adecuada en las dietas para finalizar ganado de carne. A pesar de que alrededor del mundo existen varias investigaciones en ese sentido, en Colombia los estudios acerca del desempeño y calidad de carne en bovinos en confinamiento con materias primas de bajo costo son escasos.

El objetivo de este estudio fue evaluar los efectos de diferentes niveles de inclusión de concentrado de bajo costo en dietas para toros F1 (Bos taurus $x$ Bos indicus) confinados bajo condiciones de bosque seco tropical.

\section{MATERIALES Y MÉTODOS}

Localización. El presente estudio fue realizado en la Hacienda La Paz, municipio de Tello (Huila, Colombia), entre las coordenadas geográficas $3^{\circ} 4^{\prime} 17.66^{\prime \prime} \mathrm{N}$ y $-75^{\circ} 8^{\prime} 38.09^{\prime \prime} \mathrm{O}$. El predio posee unas características bioclimáticas con una altitud de 575 m.s.n.m., promedio de temperatura $26.4^{\circ} \mathrm{C}$, humedad relativa de $71 \%$ y precipitación de $1300 \mathrm{~mm}$ año-1 y está caracterizado como zona de vida de Bosque Seco Tropical (Bs- T) (5).

Animales y tratamientos. Fueron utilizados 36 machos enteros F1 Bos taurus $x$ Bos indicus con peso inicial de $347 \pm 20 \mathrm{~kg}$ y un promedio de 18 meses de edad, los cuales fueron distribuidos aleatoriamente en los tratamientos experimentales. Previamente se realizó un examen clínico a todos los animales y desparasitados con albendazol ${ }^{\circledR}$. Previamente los animales se sometieron a un período de adaptación de 21 días antes de comenzar el periodo experimental. Cada animal fue alojado en corral individual de $3 \mathrm{~m}$ de ancho por $4.5 \mathrm{~m}$ de largo, con cerca lateral de madera atornillada y piso de cemento. Cerca del $50 \%$ del área del corral era techada y poseían comederos y bebederos lineales (tipo canoa) de $70 \mathrm{~cm}$ de longitud, $30 \mathrm{~cm}$ de profundidad y $35 \mathrm{~cm}$ de ancho en cemento. 
Los tratamientos evaluados fueron: $\mathrm{T}_{1}=85 \%$ forraje: $15 \%$ concentrado, $\mathrm{T}_{2}=75 \%$ forraje: $25 \%$ concentrado, $\mathrm{T}_{3}=65 \%$ forraje: $35 \%$ concentrado y $\mathrm{T}_{4}=55 \%$ forraje: $45 \%$ concentrado, dichas proporciones fueron tomadas teniendo en cuenta el consumo de materia seca del animal. Las dietas fueron ofrecidas dos veces al día a las 08:00 y a las 16:00 horas, los animales contaban con agua ad libitum. Como fuente de forraje se utilizó pasto Pennisetum $s p$, el cual fue establecido previamente en un área de $10 \mathrm{ha}$. El forraje se cortaba con maquinaria diariamente y se picaba a un tamaño de partícula entre $1-2 \mathrm{~cm}$. La edad de corte del forraje varió entre 45 a 50 días. El concentrado fue elaborado utilizando las materias primas y subproductos disponibles en la región entre los que se encuentra semilla de algodón (Gossypium sp.), cascarilla de algodón, torta de palmiste (Elaeis guineensis), melaza, urea, pre mezcla mineral, fosfato bicálcico y carbonato de calcio $\left(\mathrm{CaCO}_{3}\right)$ (Tabla 1 y 2 ).

Tabla 1. Composición bromatológica de los Ingredientes utilizados en las dietas experimentales.

\begin{tabular}{|c|c|c|c|c|c|c|c|c|c|c|c|}
\hline Alimento & $\begin{array}{l}\text { MS } \\
(\%)\end{array}$ & $\begin{array}{l}\text { PC } \\
(\%)\end{array}$ & $\begin{array}{c}\text { EE } \\
(\%)\end{array}$ & $\begin{array}{l}\text { MM } \\
(\%)\end{array}$ & $\begin{array}{l}\text { FDN } \\
(\%)\end{array}$ & $\begin{array}{l}\text { FDA } \\
(\%)\end{array}$ & $\begin{array}{l}\text { CNF } \\
(\%)\end{array}$ & $\begin{array}{l}\text { NDT } \\
(\%)\end{array}$ & $\begin{array}{c}\text { EM } \\
(\text { Mcal/kg) }\end{array}$ & $\begin{array}{c}\text { ENm } \\
\text { (Mcal/kg) }\end{array}$ & $\begin{array}{c}\text { ENp } \\
(\text { Mcal/kg) }\end{array}$ \\
\hline Pasto Elefante & 28.0 & 4.6 & 2.5 & 6.4 & 73.1 & 63.4 & 13.4 & 55.0 & 2.0 & 1.1 & 0.6 \\
\hline Semilla de Algodón & 91.0 & 21.2 & 17.5 & 4.4 & 46.8 & 36.9 & 10.1 & 84.5 & 3.1 & 2.1 & 1.4 \\
\hline Cáscara de algodón & 89.6 & 4.2 & 1.6 & 3.6 & 85.6 & 66.9 & 5.0 & 42.0 & 1.5 & 0.7 & 0.1 \\
\hline Harina de Arroz & 88.6 & 13.8 & 14.5 & 8.5 & 25.6 & 15.2 & 37.6 & 83.4 & 3.0 & 2.0 & 1.4 \\
\hline Palmiste & 93.7 & 14.0 & 11.7 & 3.4 & 64.6 & 55.9 & 6.3 & 62.0 & 2.2 & 1.4 & 0.8 \\
\hline Melaza & 65.3 & 4.7 & 1.5 & 8.9 & 0.0 & 0.0 & 84.9 & 72.0 & 2.6 & 1.7 & 1.1 \\
\hline Urea & 99.0 & 286.0 & 0.0 & 0.0 & 0.0 & 0.0 & 0.0 & 100.0 & 3.6 & 2.5 & 1.8 \\
\hline
\end{tabular}

MS: materia seca; PC: proteína cruda; EE: extracto etéreo; MM: materia mineral; FDN: fibra detergente neutro; FDA: fibra detergente ácido; CNF: carbohidratos no fibrosos; NDT: nutrientes digestibles totales; EM: energía metabolizable; ENm: energía neta metabolizable; ENp: energía neta de producción.

Tabla 2. Composición porcentual y bromatológica de las dietas experimentales.

\begin{tabular}{|c|c|c|c|c|}
\hline \multirow{2}{*}{ Alimento } & \multicolumn{4}{|c|}{ Tratamientos } \\
\hline & 1 & 2 & 3 & 4 \\
\hline Pennisetum purpureum & 85.00 & 75.00 & 65.00 & 55.00 \\
\hline Semilla de Algodón & 3.90 & 6.50 & 9.10 & 11.70 \\
\hline Cáscara de algodón & 2.40 & 4.00 & 5.60 & 7.20 \\
\hline Harina de Arroz & 4.05 & 6.75 & 9.45 & 12.15 \\
\hline Palmiste & 3.30 & 5.50 & 7.70 & 9.90 \\
\hline Melaza & 0.90 & 1.50 & 2.10 & 2.70 \\
\hline Urea & 0.15 & 0.25 & 0.35 & 0.45 \\
\hline Sal Blanca & 0.09 & 0.15 & 0.21 & 0.27 \\
\hline carbonato de Calcio & 0.12 & 0.20 & 0.28 & 0.36 \\
\hline Fosfato Bicálcico & 0.05 & 0.08 & 0.11 & 0.14 \\
\hline Mineral premix ${ }^{1}$ & 0.05 & 0.08 & 0.11 & 0.14 \\
\hline \multicolumn{5}{|l|}{ Composición } \\
\hline PC (\%) & 6.33 & 7.48 & 8.64 & 9.79 \\
\hline EE (\%) & 3.83 & 4.72 & 5.61 & 6.50 \\
\hline FDN $(\%)$ & 69.18 & 66.57 & 63.96 & 61.35 \\
\hline FDA $(\%)$ & 59.30 & 56.80 & 54.90 & 51.30 \\
\hline CNF (\%) & 14.40 & 15.06 & 15.73 & 16.40 \\
\hline NDT (\%) & 57.28 & 58.79 & 60.31 & 61.83 \\
\hline EM (Mcal/kg) & 2.07 & 2.13 & 2.18 & 2.24 \\
\hline ENm (Mcal/kg) & 1.22 & 1.27 & 1.31 & 1.36 \\
\hline ENp (Mcal/kg) & 0.65 & 0.69 & 0.74 & 0.78 \\
\hline
\end{tabular}


El alimento fue calculado permitiendo entre 5 a $10 \%$ de rechazos, siendo pesado tanto el concentrado como forraje diariamente. Para determinar el consumo de materia seca (CMS) se tenían en cuenta el alimento ofrecido y se descontaban los rechazos de cada animal al día siguiente antes de la primera alimentación para ajustar el suministro de alimento diario.

Análisis Bromatológicos. Las muestras de forraje y de los concentrados utilizados en las dietas experimentales fueron colectadas semanalmente, empacadas en bolsas plásticas y congeladas para posteriores análisis. La composición bromatológica de las dietas se realizó de acuerdo a los métodos establecidos por la AOAC (6) para materia seca (MS), materia orgánica (MO), proteína cruda (PC), extracto etéreo ( $E E)$ y cenizas. La fibra en detergente neutro (FDN) y fibra en detergente ácida (FDA) según Van Soest et al (7). Los carbohidratos no fibrosos se calcularon de acuerdo a Sniffen et al, (8) y los valores de nutrientes digestibles totales (NDT), energía metabolizable (EM), energía neta de mantenimiento $(E N m)$ y energía neta de producción (ENp) se tomaron teniendo en cuenta los aportes de energía de los alimentos del NRC para ganado de carne (9) y a Valadares et al (10).

Desempeño y morfometría de la canal. La ganancia diaria de peso (GDP) se determinó pesando los animales cada 30 días, durante 6 períodos consecutivos utilizando una balanza electrónica. Los pesajes se realizaron en la mañana antes de la primera alimentación. La conversión alimentar (CA) se calculó con base en la ecuación:

$C A=(G D P / C M S) \times 100$.

Una vez los animales alcanzaron un peso igual o superior a $450 \mathrm{~kg}$, fueron transportados a un frigorífico comercial (CEAGRODEX, Rivera Huila). Los animales se dejaron en cuarentena por 15 horas (h) con acceso a agua ad libitum. Posteriormente, se realizó inspección veterinaria y se pasaron al área de insensibilización donde fueron aturdidos con pistola de perno cautivo (11), inmediatamente se izaron y se realizó proceso de faenado. Se pesaron las canales, se dividieron con sierra de cinta, se lavaron y se etiquetaron. La determinación del peso de la canal caliente (PCC) se realizó una vez finalizado el proceso de faenado y se expresó en kg, mientras que el rendimiento en canal se expresó en porcentajes dado por el peso del canal en relación con el peso en pie.
Las medidas morfométricas se tomaron a las $12 \mathrm{~h}$ post-mortem, utilizando cinta métrica, siguiendo la metodología del ICTA (12) se midió el perímetro de la pierna $(P P)$, la longitud de canal $(L C)$, la longitud de la pierna $(L P)$, espesor de la pierna $(E P)$, ancho del pecho $(A P)$, ancho del lomo $(A L)$ y largo del lomo $(L M)$.

Calidad de carne. Previo al almacenamiento en cuarto frio $\left(-4^{\circ} \mathrm{C}\right)$ se realizaron la toma de muestras de $\mathrm{pH}$ y temperatura. Para la medición de $\mathrm{pH}$ se siguieron los parámetros de la metodología de Honikel (13), se usó potenciómetro digital portátil Hanna ${ }^{\circledR}$. La temperatura se midió con termómetro portátil digital, marca Multi - Thermometer ${ }^{\circledR}$. Los datos se tomaron del Longissimus dorsi $(L D)$ en 3 puntos diferentes. La toma de $\mathrm{pH}$ y temperatura se repitió, a las 3, 6, 12, 24 y 48 h post-mortem.

En cuanto al color se tomaron muestras del músculo $L D$, a la altura de las vértebras torácicas 12 y 13 a las $12 \mathrm{~h}$ post-mortem. Las muestras fueron aisladas con papel aluminio, refrigeradas y llevadas al laboratorio de postcosecha de la Facultad de Ingeniería Agronómica de la Universidad del Tolima. Las muestras se analizaron después a las $24 \mathrm{~h}$ y $48 \mathrm{~h}$ postmortem. La medición del color fue evaluada mediante un espectrofotómetro Minolta modelo CM con iluminación estándar D65 y una apertura de $2.54 \mathrm{~cm}$. Para el procedimiento se siguió el sistema CIE L*a*b incorporado en el espectrofotómetro. El $L D$ fue diseccionado por la mitad con el fin de obtener porciones similares, seguidamente se realizaron 5 tomas fotográficas y se obtuvieron los valores de las tres coordenadas. Finalmente se obtuvo el promedio de cada una de las coordenadas para establecer la medida final.

La textura fue evaluada por el método Cizalla de Warner-Bratzler. Utilizando un texturómetro, se determinó la dureza del músculo cocinado, mediante el registro del esfuerzo máximo para cortar la muestra. Las muestras se cocinaron en una parrilla eléctrica, hasta alcanzar una temperatura interna de $70^{\circ} \mathrm{C}$. Posteriormente, las muestras se enfriaron dentro de una bolsa en una cámara a $5^{\circ} \mathrm{C}$ durante $24 \mathrm{~h}$. Una vez frías se cortaron en prismas de $1 \mathrm{~cm}$ de ancho por 3 $\mathrm{cm}$ de largo.

Análisis del perfil lipídico. Para determinar los ácidos grasos se utilizó la técnica de Folch (14) sobre 16 muestras de $100 \mathrm{~g}$ del músculo $L D$ en frío. El proceso consistió en homogenizar, extraer 
con solución Folch, filtrar, separar y evaporar los solventes para recuperar la grasa del hexano; luego de obtener la grasa sin sus componentes, se llevó a cabo la técnica de saponificación para liberar los ácidos grasos, metilación y por último la cuantificación de los ácidos grasos por cromatografía de gases (15).

Metabolitos sanguíneos. Se tomaron 4 muestras sanguíneas de cada animal en los últimos cuatro periodos de muestreo. La colecta de las muestras se hizo previo ayuno de alimento y se realizó a través de venopunción coccígea utilizando tubos BD Vacutainer sin anticoagulante. Una vez tomadas se enviaron bajo refrigeración al Laboratorio de Diagnóstico Veterinario - LADIVET, perteneciente a la Universidad del Tolima. Las muestras de sangre fueron centrifugadas a $2000 \mathrm{rpm}$ por 10 minutos, el suero fue refrigerado y almacenado a $-20^{\circ} \mathrm{C}$. Posteriormente, se determinó nitrógeno ureico sanguíneo (NUS), glucosa, beta-hidroxibutirato (BHB), triglicéridos y fósforo mediante el método de espectrometría utilizando kits comerciales y el equipo analizador semiautomático BTS-350 BioSystems ${ }^{\circledR}$.

Análisis estadístico. Se realizó un análisis de variancia (ANOVA) con una confiabilidad del $95 \%$ y una comparación de medias de cada uno de los tratamientos por medio de un test de
Tukey para todas las variables. Los datos fueron analizados por medio del software estadístico SAS. Igualmente, se realizó un análisis de regresión lineal (L) y cuadrática (Q) para las variables donde fueron observadas diferencias significativas.

\section{RESULTADOS}

Desempeño animal y parámetros de la canal. La GDP y el peso final de sacrificio aumentó a medida que la inclusión del concentrado en la dieta se incrementó $(p<0.05)$, pasando de 0.55 $\mathrm{kg}$ para $0.67 \mathrm{~kg}$ y de $450 \mathrm{~kg}$ para $468 \mathrm{~kg}$ para los tratamientos con $15 \%$ y $45 \%$ de concentrado, respectivamente (Tabla 3 ). Sin embargo, el CMS, CA y el RC no se vieron afectados por los niveles de concentrado en la dieta $(p>0.05)$. Igualmente, no se observaron diferencias en los parámetros morfométricos de la canal $(p>0.05)$.

Calidad de la carne. Los principales parámetros de determinación de calidad: temperatura de la canal $\left({ }^{\circ} \mathrm{C}\right)$, textura $(\mathrm{kg} \mathrm{cm}-2), p H$ a las 0 y $48 \mathrm{~h}$, no presentaron diferencias entre los tratamientos $(p>0.05)$. Sin embargo, hubo diferencias $(p<0.05)$ en el color de la carne, en la coordenada $L(p<0.05)$, observando un aumento a medida que la proporción del concentrado en la dieta se incrementaba (Tabla 4).

Tabla 3. Desempeño y Parámetros de la Canal en toros F1 (Bos Taurus $x$ Bos indicus) suplementados con diferentes niveles de Concentrado de bajo costo.

\begin{tabular}{|c|c|c|c|c|c|c|c|}
\hline \multirow{2}{*}{ Ítem } & \multicolumn{4}{|c|}{ Tratamientos } & \multirow{2}{*}{ EPM1 } & \multicolumn{2}{|c|}{ p - valor } \\
\hline & 1 & 2 & 3 & 4 & & $\mathbf{L}$ & $\mathbf{Q}$ \\
\hline Peso inicial, $\mathrm{kg}$ & 348.5 & 347.3 & 347.6 & 350.0 & 2.038 & 0.945 & 0.853 \\
\hline Peso final, $\mathrm{kg}$ & $450.0^{c}$ & $456.5^{\mathrm{bc}}$ & $458.5^{b}$ & $468.0^{\mathrm{a}}$ & 1.620 & 0.001 & 0.855 \\
\hline$G D P, \mathrm{~kg} \mathrm{~d}^{-1}$ & $0.55^{b}$ & $0.56^{b}$ & $0.63^{a}$ & $0.67^{a}$ & 0.019 & 0.045 & 0.663 \\
\hline$C M S, \mathrm{~kg} \mathrm{~d}^{-1}$ & 7.00 & 6.56 & 7.62 & 7.27 & 0.116 & 0.912 & 0.832 \\
\hline$C A$ & 13.06 & 11.87 & 12.22 & 10.94 & 0.304 & 0.748 & 0.931 \\
\hline RC (\%) & 51.05 & 50.99 & 52.15 & 52.34 & 0.255 & 0.873 & 0.802 \\
\hline \multicolumn{8}{|c|}{ Parámetros de la canal $(\mathrm{cm})$} \\
\hline Longitud de la canal & 144.1 & 140.5 & 137.9 & 140.9 & 17.95 & 0.217 & 0.434 \\
\hline Perímetro de la pierna & 101.4 & 103.3 & 103.5 & 104.4 & 12.83 & 0.498 & 0.691 \\
\hline Ancho del lomo & 14.9 & 14.8 & 14.8 & 14.6 & 0.85 & 0.927 & 1.000 \\
\hline Largo del lomo & 86.3 & 86.1 & 84.8 & 82.3 & 22.3 & 0.699 & 0.475 \\
\hline
\end{tabular}

${ }^{1}$ EPM: Error padrón de la media. Diferencias entre tratamientos en la fila se fueron identificadas con letras diferentes. 
Tabla 4 Parámetros de la Calidad de la Carne de toretes $\mathrm{F} 1$ (Bos Taurus $x$ Bos indicus) alimentados con diferentes niveles de concentrado de bajo costo.

\begin{tabular}{|c|c|c|c|c|c|c|}
\hline \multirow{2}{*}{ Ítem } & \multicolumn{4}{|c|}{ Tratamientos } & \multirow{2}{*}{ EPM $^{1}$} & \multirow{2}{*}{ P valor } \\
\hline & 1 & 2 & 3 & 4 & & \\
\hline $\mathrm{Temp}^{2}\left({ }^{\circ} \mathrm{C}\right)$ & 5.80 & 5.80 & 6.00 & 6.10 & 0.20 & 0.343 \\
\hline $\begin{array}{l}\text { Textura } \\
\left(\mathrm{kg} \mathrm{cm}^{-2}\right)\end{array}$ & 6.76 & 8.16 & 6.38 & 7.34 & 4.80 & 0.405 \\
\hline $\mathrm{pHOh}$ & 6.54 & 6.81 & 6.49 & 6.56 & 0.26 & 0.598 \\
\hline$p H 48 \mathrm{~h}$ & 5.78 & 6.09 & 5.86 & 5.93 & 0.18 & 0.519 \\
\hline \multicolumn{7}{|l|}{ Color } \\
\hline L & $27.40^{\mathrm{b}}$ & $29.08^{b}$ & $30.28^{\mathrm{ab}}$ & $32.58^{\mathrm{a}}$ & 6.09 & 0.050 \\
\hline A & 14.14 & 13.55 & 14.16 & 14.81 & 2.45 & 0.470 \\
\hline b & 3.14 & 2.86 & 2.95 & 3.58 & 1.24 & 0.591 \\
\hline
\end{tabular}

${ }^{1}$ EPM: Error padrón de la media; Temp2: Temperatura de la canal 12 horas después del sacrificio. Color rojo 645700 nm; color amarillo 578, 580 o 582 nm. Diferencias entre tratamientos en la fila fueron identificadas con letras diferentes.
Perfil lipídico. Hubo diferencias en los ácidos grasos saturados $(p<0.05)$, caprílico, cáprico y tridecanoico en los cuales se presentó una disminución a medida el nivel de inclusión del concentrado en la dieta aumentó (Tabla 5). Sin embargo, la proporción de los ácidos grasos insaturados y poliinsaturados no presentaron diferencias entre los tratamientos $(p>0.05)$.

Perfil sanguíneo. No se presentaron diferencias $(p>0.05)$ entre ninguno de los tratamientos para los diferentes metabolitos en sangre (Tabla 6).

Tabla 5. Perfil de ácidos grasos de la carne de toretes F1 (Bos Taurus $x$ Bos indicus) mestizos suplementados con diferentes niveles de concentrado en $\mathrm{g}$ por $100 \mathrm{~g}$ de músculo.

\begin{tabular}{|c|c|c|c|c|c|c|c|}
\hline \multirow{2}{*}{ Ácidos Grasos } & \multicolumn{4}{|c|}{ Tratamientos } & \multirow{2}{*}{ EPM 1} & \multicolumn{2}{|c|}{ p - value } \\
\hline & 1 & 2 & 3 & 4 & & $\mathbf{L}$ & $\mathbf{Q}$ \\
\hline \multicolumn{8}{|l|}{ SATURADOS } \\
\hline 6:0 Caproico & 0.063 & 0.05 & 0.024 & 0.028 & 0.0004 & 0.190 & 0.450 \\
\hline 8:0 Caprílico & $0.063^{a}$ & $0.05^{\mathrm{ab}}$ & $0.024^{b}$ & $0.028^{b}$ & 0.0004 & 0.009 & 0.449 \\
\hline 10:0 Cáprico & $0.073^{a}$ & $0.066^{\mathrm{ab}}$ & $0.061^{\mathrm{ab}}$ & $0.049^{b}$ & 0.0004 & 0.001 & 0.760 \\
\hline 12:0 Láurico & 1.428 & 1.240 & 1.489 & 1.722 & 0.158 & 0.362 & 0.243 \\
\hline 13:0 Tridecanoico & $0.063^{a}$ & $0.054^{\mathrm{ab}}$ & $0.031^{\mathrm{b}}$ & $0.034^{b}$ & 0.0003 & 0.001 & 0.440 \\
\hline 14:0 Mirístico & 8.812 & 8.269 & 8.968 & 9.845 & 1.054 & 0.238 & 0.132 \\
\hline 16:0 Palmítico & 32.276 & 31.471 & 31.869 & 32.697 & 0.807 & 0.217 & 0.237 \\
\hline 18:0 Esteárico & 18.528 & 20.896 & 18.883 & 20.040 & 10.035 & 0.436 & 0.371 \\
\hline 20:0 Araquídico & 0.175 & 0.225 & 0.224 & 0.188 & 0.003 & 0.077 & 0.053 \\
\hline 22:0 Behénico & 0.091 & 0.104 & 0.075 & 0.065 & 0.0004 & 0.711 & 0.857 \\
\hline 23:0 Tricosanoico & 0.084 & 0.107 & 0.059 & 0.046 & 0.0009 & 0.510 & 0.423 \\
\hline 24:0 Tetracosanoic & 0.112 & 0.232 & 0.146 & 0.189 & 0.006 & 0.634 & 0.678 \\
\hline \multicolumn{8}{|l|}{ MONOINSATURADOS } \\
\hline 14:1n5 - Miristoleico & 1.538 & 0.899 & 1.389 & 1.245 & 0.168 & 0.257 & 0.170 \\
\hline 15:1 - Pentadecanoico & 0.866 & 0.906 & 0.903 & 0.796 & 0.034 & 0.723 & 0.543 \\
\hline 16:1n7 - Palmitoleico & 3.850 & 3.194 & 3.632 & 3.106 & 0.616 & 0.068 & 0.110 \\
\hline 17:1n9Heptadecanoico & 0.994 & 1.047 & 0.985 & 0.965 & 0.010 & 0.627 & 0.662 \\
\hline 18:1n9t Elaídico & 0.721 & 0.718 & 0.820 & 1.014 & 0.052 & 0.071 & 0.076 \\
\hline 18:1n9c oleico & 29.123 & 28.227 & 28.606 & 25.892 & 11.075 & 0.156 & 0.242 \\
\hline 20:1n9 Eicosenoico & 0.091 & 0.072 & 0.077 & 0.074 & 0.0002 & 0.158 & 0.292 \\
\hline \multicolumn{8}{|l|}{ POLIINSATURADOS } \\
\hline 18:2n6c Linoléico & 0.865 & 1.715 & 1.415 & 1.518 & 0.225 & 0.522 & 0.291 \\
\hline 18:3n3 Linolénico & 0.104 & 0.097 & 0.082 & 0.097 & 0.003 & 0.438 & 0.231 \\
\hline 20:4n6 Araquidónico & 0.081 & 0.356 & 0.24 & 0.361 & 0.020 & 0.289 & 0.348 \\
\hline Saturados & 63.453 & 64.523 & 63.706 & 66.714 & 17.650 & 0.749 & 0.607 \\
\hline Insaturados & 35.722 & 34.520 & 34.520 & 32.259 & 17.902 & 0.562 & 0.333 \\
\hline Poliinsaturados & 0.721 & 0.718 & 0.820 & 1.014 & 0.052 & 0.758 & 0.609 \\
\hline
\end{tabular}

${ }^{1}$ EPM: Error padrón de la media. Diferencias entre tratamientos en la fila fueron identificadas con letras diferentes. 
Tabla 6. Parámetros sanguíneos en bovinos F1 (Bos Taurus $x$ Bos indicus) alimentados con diferentes niveles de concentrado de baja calidad.

\begin{tabular}{lcccccc}
\hline \multirow{2}{*}{\multicolumn{1}{c}{ Ítem }} & \multicolumn{9}{c}{ Tratamientos } & \multirow{2}{*}{ EPM1 P valor } \\
\cline { 2 - 6 } & $\mathbf{1}$ & $\mathbf{2}$ & $\mathbf{3}$ & $\mathbf{4}$ & & \\
\hline NUS (mg/dL) & 5.41 & 6.33 & 6.84 & 7.11 & 6.42 & 0.218 \\
Glucosa (mg/dL) & 73.78 & 74.78 & 76.56 & 73.69 & 60.9 & 0.984 \\
Colesterol (mg/dL) & 196.5 & 214.1 & 225.2 & 199.5 & 148.9 & 0.107 \\
Triglicéridos (mg/dL) & 44 & 46.06 & 43.06 & 41.31 & 12.69 & 0.666 \\
Fósforo (mg/dL) & 8.23 & 8.26 & 7.76 & 7.87 & 0.60 & 0.142 \\
BHB (mmol/dL) & 0.37 & 0.4 & 0.34 & 0.32 & 0.01 & 0.119 \\
\hline
\end{tabular}

${ }^{1}$ EPM: Error padrón de la media.

\section{DISCUSIÓN}

Cuando los alimentos contienen una fracción alta de FDN y FDA existe bajo consumo y baja digestibilidad, dietas ricas en fibra, no permiten una liberación de nutrientes por parte de los microorganismos debido a las altas fracciones indigestibles (16). Cuando esto ocurre hay una activación hormonal, en especial la leptina y la grelina, relacionada con la saciedad y una disminución en el consumo generando una influencia inversa entre consumo y ganancia de peso (17). Lo anterior puede explicar el comportamiento en el consumo y ganancias de peso de este estudio, los cuales son bajos cuando comparados con estudios utilizando dietas con menores cantidades de FDN y FDA. Resultados similares a este estudio fueron obtenidos por (18), quienes al alimentar novillos mestizos con dietas que contenían 5.35 y $65 \%$ de concentrado, observaron valores de CMS entre 6.6 y $8.1 \mathrm{~kg}$ $\mathrm{d}^{-1}$. Entre tanto, y como era de esperar a medida que aumentó el concentrado en la dieta mejoró la GPD. Entre tanto Alonso et al (19) alimentaron bovinos con ensilaje de Brachiaria brizantha $\mathrm{CV}$. Marandu $y$ concentrado en las relaciones $\mathrm{F}: \mathrm{C}$ de $80: 20,65: 35,50: 50,65: 35$ y reportaron consumos de MS de 5.31, 6.43, 8.13 y 9.52 $\mathrm{kg} \mathrm{d}^{-1}$. La divergencia de resultados entre los trabajos permite inferir que el CMS, puede estar afectado por diversos factores del animal, del alimento y de las condiciones climáticas que interactúan.

Con relación a los parámetros de la canal, puede observarse que están dentro de los parámetros reportados para razas cebuínas (20). La longitud de la canal fue una variable afectada estadísticamente. No se le puede atribuir esa diferencia a la dieta directamente y si a la variabilidad genética dentro de los animales del estudio, ya que las diferencias morfológicas son parámetros de alta heredabilidad (21), a pesar que los animales se seleccionaron para obtener grupos homogéneos. De este modo, la influencia dada por el desempeño propio del animal queda relegada frente a la relevancia de los factores asociados con la genética del animal y su influencia en la conformación de la canal (22).

Los parámetros de calidad de carne en este estudio no están dentro de los valores esperados para este tipo de experimento (23). Al analizar la temperatura a las $12 \mathrm{~h}$ se puede observar que ninguna de las medidas son las ideales para lograr detener los procesos bacterianos, pudiendo esto afectar la maduración del músculo. Es importante garantizar que la temperatura de la canal tenga una caída cercana a los $5^{\circ} \mathrm{C}$ durante las primeras $12 \mathrm{~h}$ post-mortem debido a que va a incidir directamente en el descenso del $\mathrm{pH}$ y en la dilución de las fibras musculares para obtener una adecuada maduración en la carne (24). Al observar los resultados de $\mathrm{pH}$ a las 48 $\mathrm{h}$ se puede apreciar un aumento de este valor $\mathrm{y}$ como existe una relación directa con los valores de temperatura a las $12 \mathrm{~h}$. La interconexión entre la temperatura y $\mathrm{pH}$ es un modelo que predice la afectación que puede tener uno u otro factor cuando son comparados entre sí y su correlación directa positiva (25). Estas mudanzas en los indicadores, pueden también estar relacionados con alteraciones en el color debido a que se aumenta o disminuye la capacidad de retención de agua en el músculo y la transformación del músculo en carne.

Al considerar los hallazgos en los parámetros referentes al color, como se ve reflejado en la diferencia encontrada en la luminosidad de la carne (coordenada $L$ ) como en el bajo valor para la tonalidad amarilla (coordenada $b$ ), se puede apreciar que la carne presentó una tonalidad un poco oscura comparada con lo referenciado en la literatura (26). Los cambios en las tonalidades pueden ser incidencia de diferentes factores como el contenido de grasa intramuscular, el estrés animal antes del sacrificio y algunos problemas relacionados con la maduración. Una de las principales causas para que el valor $L$ sea inferior es lo relacionado con la edad del animal. Cuando se obtiene carne de un animal con edad mayor por lo general la capacidad de oxigenación celular disminuye y la mioglobina presente no posee una cantidad suficiente de oxígeno por lo cual la energía del músculo es insuficiente para el rompimiento de las fibras musculares, aumentando la retención de agua (27). Cuando el animal es sometido a estrés 
antes del sacrificio, la maduración de la carne es afectada, por lo tanto, se puede inferir que durante el proceso de transporte $y / o$ sacrificio de los animales fueron afectados por estrés, lo cual afecto los resultados de este estudio.

La cantidad de ácidos grasos reportados en este estudio concuerda con los resultados presentados por otros trabajos en este tipo cruce racial (28). Cabe destacar que los resultados reportados por la literatura indican una mínima influencia de la dieta en el perfil de ácidos grasos en la carne de rumiantes, por lo que los cambios en los diferentes ácidos no son mayores al $5 \%$ $(29,30)$. Los ácidos grasos saturados son ácidos originados principalmente por el metabolismo de bacterias y levaduras presentes en el ambiente ruminal (31) y se manifiestan en la leche y carne. Cuando el animal es alimentado con una mayor cantidad de fibra las condiciones ruminales, principalmente el $p H$, van a ser propicio para producir una mayor cantidad de acetato y ocurre aumento de los ácidos grasos saturados (32). Esto puede explicar en parte la variabilidad mostrada en los ácidos grasos de las dietas con menor nivel de concentrado en este estudio. El ácido tricosanoico, aunque posee el mismo comportamiento metabólico de los otros ácidos saturados que presentaron diferencia estadística, puede tener una mayor deposición debido a que es formada por la síntesis de novo (33). Por ende, la deposición de los ácidos grasos presentes en la carne va a depender del metabolismo realizado por la microbiota ruminal y la producción de los ácidos grasos volátiles en el rumen. Estos mismos factores afectan los contenidos de los ácidos grasos poliinsaturados en la carne.

Los metabolitos sanguíneos dependen del metabolismo del animal y sus deficiencias o excesos de nutrientes en la dieta. Esta interpretación de los valores va a ser primordial para poder realizar identificación de enfermedades asociadas con disturbios metabólicos o también puede distinguir disturbios manejables a través de la dieta para mejorar el desempeño del animal (34). Otro factor que refleja esta condición en el balance o desbalance entre la proteína y la energía en la dieta son los resultados generados por el NUS. En este estudio se puede inferir que dichas proporciones fueron ligeramente bajas. En los tratamientos evaluados fueron observados valores de colesterol sanguíneo elevados, esto podría ser explicado por la presencia de torta de palmiste en la dieta. La torta de palmiste contiene gran cantidad de ácido palmítico, que al ser metabolizado por el animal puede encontrarse de manera libre en sangre y representar un porcentaje relativamente alto (35).

En conclusión, el aumento en la proporción de concentrado elaborado con subproductos agrícolas producidos en el departamento del Huila, mejora la ganancia de peso y el peso final de sacrificio en toros F1 (Bos Taurus $x$ Bos indicus) confinados en condiciones de bosque seco tropical durante la fase de finalización. La Temperatura, textura y $\mathrm{pH}$ de la carne y los metabolitos sanguíneos en los animales no cambian cuando se incrementa los niveles de concentrado en los toros. El parámetro $L$ del color de la carne aumenta cuando se incrementa la proporción de concentrado y la proporción de los ácidos grasos saturados cáproico, caprílico y tridecanoico disminuyen cuando se incrementan los niveles de concentrado en la dieta de los bovinos mestizos confinados.

\section{Conflicto de interés}

Los autores declaran ningún conflicto de interés.

\section{REFERENCIAS}

1. Ruviaro C, da Costa J, Florindo T, Rodrigues W, de Medeiros G, Vasconcelos P. Economic and environmental feasibility of beef production in different feed management systems in the Pampa biome, southern Brazil. Ecological indicators. 2016; 60:930-939. https://doi. org/10.1016/j.ecolind.2015.08.042
2. Callejas-Juárez N, Rebollar-Rebollar S, Ortega-Gutiérrez J, Domínguez-Viveros J. Parámetros bio-económicos de la producción intensiva de la carne de bovino en México. Rev Mex Cienc Pecuarias. 2017; 8(2):129138. https://doi.org/10.22319/rmcp. v8i2.4415 
3. Fidelis $\mathrm{H}$, Bonilha $\mathrm{S}$, Tedeschi $\mathrm{L}$, Branco $\mathrm{R}$, Cyrillo J, Mercadante M. Residual feed intake, carcass traits and meat quality in Nellore cattle. Meat Sci. 2017; 128:34-39. https:// doi.org/10.1016/j.meatsci.2017.02.004

4. Oliver L, Sánchez R. Las cuatro grandes empresas comercializadoras y los precios internacionales de los alimentos. Economía Informa. 2016; 400:24-39. https://doi. org/10.1016/j.ecin.2016.09.003

5. Pizano C, García H. El bosque seco tropical en Colombia. Instituto de Investigación de Recursos Biológicos Alexander Von Humboldt, Bogotá (Colombia). Ministerio de Ambiente y Desarrollo Sostenible, Bogotá (Colombia) 2014. http://repository.humboldt.org.co/ handle/20.500.11761/9333

6. AOAC. Official Methods of Analysis Association of Official Analytical Chemists. Version 15 edition. Arlington, VA; 2019. https://www.aoac.org/official-methods-ofanalysis-21st-edition-2019/

7. Van Soest PJ, Robertson JB, Lewis BA. Methods for Dietary Fiber, Neutral Detergent Fiber, and Nonstarch Polysaccharides in Relation to Animal Nutrition. J Dairy Sci. 1991; 74(10):3583-3597. https://doi. org/10.3168/jds.S0022-0302(91)78551-2

8. Sniffen CJ, O'Connor JD, Van Soest PJ, et al. A net carbohydrate and protein system for evaluating cattle diets: II. Carbohydrate and protein availability. J Anim Sci. 1992; 70(11):3562-3577. https://doi. org/10.2527/1992.70113562x

9. NRC. Nutrient requirements of beef cattle. National Academies of Sciences, Engineering, and Medicine. National Academies Press; 2016. https://www.nap.edu/catalog/19014/ nutrient-requirements-of-beef-cattleeighth-revised-edition

10. Valadares S, Silva L, Gionbelli M, Rotta P, Marcondes M, Chizzotti M, Prados L. Nutrient requirements of Zebu and crossbred cattle (BR-CORTE). Universidade Federal de Viçosa: Brasil; 2016. https://v3.brcorte. com.br/bundles/junglebrcorte2/book2016/ en/c0.pdf

11. Linares CP. Cambios en las prácticas de manejo antes y durante el sacrificio para disminuir la presencia de carne DFD en bovinos. Nacameh. 2011; 5(1):5968. https://dialnet.unirioja.es/servlet/ articulo?codigo $=4024343$
12. Amador GI, Palacios GA, Maldonado CMA. Sistema ICTA de Clasificación de Canales y Cortes de Carne Bovina. Bogotá D.C; 1995. https://books.google.com.co/books?id=PW $\underline{x d 1 b S q A I I C \& p r i n t s e c}=$ frontcover\&hl=es\#v =onepage\&q\&f=false

13. Honikel K. Reference methods for the assessment of physical characteristics of meat. Meat Sci. 1998; 49:447-457. https:// doi.org/10.1016/S0309-1740(98)00034-5

14. Folch J, Lee M, Sloane Stanley G. A simple method for the isolation and purification of total lipids from animal tissues. J Biol Chem. 1957; 226(1):497-509. https://www.jbc. org/article/S0021-9258(18)64849-5/pdf

15. Al-Bukhaiti WQ, Noman A, Qasim AS, AlFarga A. Gas chromatography: Principles, advantages and applications in food analysis. Internat J Agric Innovat Research. 2017; 6(1):123-128. https://ijair.org/ administrator/components/com jresearch/ files/publications/IJAIR 2467 FINAL.pdf

16. McGrath J, Duval S, Tamassia L, Kindermann M, Stemmler R, de Gouvea V, Celi P. Nutritional strategies in ruminants: $A$ lifetime approach. Res Vet Sci. 2018; 116:28-39. https://doi.org/10.1016/j. rvsc.2017.09.011

17. Di Lorenzo N, Smith D, Quinn M, May M, Ponce C, Steinberg W, Galyean M. Effects of grain processing and supplementation with exogenous amylase on nutrient digestibility in feedlot diets. Livest Sci. 2011; 137(13):178-184. https://doi.org/10.1016/j. livsci.2010.11.003

18. Da Silva GS, Véras AS, de Andrade M, Dutra WM, Neves ML, Souza EJ, de Lima DM. Performance and carcass yield of crossbred dairy steers fed diets with different levels of concentrate. Trop Anim Health Pro. 2015; 47(7):1307-1312. https://doi.org/10.1007/ s11250-015-0864-X

19. Alonso MP, Moraes EH, Pina DD, Pereira DH, Mombach MA, Gimenez B, Wruck FJ. Suplementação concentrada para bovinos de corte em sistema de integração lavoura e pecuária no período das águas. Rev Bras Saúde Prod Anim. 2013; 15(2):339349. http://dx.doi.org/10.1590/S1519$\underline{99402014000200018}$ 
20. Abdelhadi OM, Babiker SA, Kijora C. Estimation of zebu cattle carcass weight using body measurements. Livestock Research Rural Develop. 2011; 23:12. http://www. Irrd.org/Irrd23/1/abde23012.htm

21. De Oliveira M, de Souza K, Vital A, Guerrero A, Valero M, Kempinski $E$, do Prado I. Clove and rosemary essential oils and encapsuled active principles (eugenol, thymol and vanillin blend) on meat quality of feedlot-finished heifers. Meat Sci. 2017; 130:50-57. https://doi.org/10.1016/j. meatsci.2017.04.002

22. Lu D, Sargolzaei M, Kelly M, Vander G, Wang Z, Mandell I, Miller SP. Genome-wide association analyses for carcass quality in crossbred beef cattle. BMC Genet. 2013; 14(1):80. https://doi.org/10.1186/1471$\underline{2156-14-80}$

23. Lozano M, Medina R, Mayorga K, García M, Ovando M, Ngapo T, Maldonado F. Effect of an allostatic modulator on stress blood indicators and meat quality of commercial young bulls in Mexico. Meat Sci. 2015; 105:63-67. https://doi.org/10.1016/j. meatsci.2015.03.012

24. Asimwe L, Kimambo A, Laswai G, Mtenga, $L$, Weisbjerg M, Madsen J. Effect of days in feedlot on growth performance, carcass and meat quality attributes of Tanzania shorthorn zebu steers. Trop Anim Health Pro. 2015; 47(5):867-876. https://doi. org/10.1016/j.meatsci.2013.04.008

25. Van de Ven, R., Pearce K, Hopkins D. Postmortem modelling of $\mathrm{pH}$ and temperature in related lamb carcasses. Meat Sci. 2014; 96(2):1034-1039. https://doi. org/10.1016/j.meatsci.2012.10.001

26. Barcellos V, Mottin C, Passetti R, Guerrero A, Eiras C, Prohman PE, Prado I. Carcass characteristics and sensorial evaluation of meat from Nellore steers and crossbred Angus vs. Nellore bulls. Acta Sci-Anim Sci. 2017; 39(4):437-448. http://dx.doi. org/10.4025/actascianimsci.v39i4.36692

27. Pearce KL, Rosenvold K, Andersen HJ, Hopkins DL. Water distribution and mobility in meat during the conversion of muscle to meat and ageing and the impacts on fresh meat quality attributes - A review. Meat Sci. 2011; 89(2):111-124. https://doi. org/10.1016/j.meatsci.2011.04.007
28. Rivaroli D, Guerrero A, Valero M, Zawadzk F, Eiras C, del Mar Campo, M, do Prado I. Effect of essential oils on meat and fat qualities of crossbred young bulls finished in feedlots. Meat Sci. 2016; 121:278-284. https://doi. org/10.1016/j.meatsci.2016.06.017

29. Shingfield KJ, Bonnet M, Scollan ND. Recent developments in altering the fatty acid composition of ruminant-derived foods. Animal. 2013; 7(s1):132-162. https://doi. org/10.1017/S1751731112001681

30. Freitas $T$, Felix $T$, Pedreira $M$, Silva $R$, Silva $F$, Silva $H$, Moreira B. Effects of increasing palm kernel cake inclusion in supplements fed to grazing lambs on growth performance, carcass characteristics, and fatty acid profile. Anim Feed Sci Tech. 2017; 226:71-80. https://doi.org/10.1016/j. anifeedsci.2017.02.009

31. De Araujo W, Leitão, R, Gehring T, Angenent $L$, Santaella $S$. Anaerobic fermentation for $\mathrm{n}$-caproic acid production: A review. Process Biochem. 2017; 54:106-119. https://doi. org/10.1016/j.procbio.2016.12.024

32. Bhatt RS, Sahoo A, Karim SA, Agrawal AR. Effects of calcium soap of rice bran oil fatty acids supplementation alone and with DL-a-tocopherol acetate in lamb diets on performance, digestibility, ruminal parameters and meat quality. J Anim Physiol An N. 2016; 100(3):578-589. https://doi. org/10.1111/jpn.12370

33. Yurchenko S, Sats A, Tatar V, Kaart T, Mootse $H$, Jõudu I. Fatty acid profile of milk from Saanen and Swedish Landrace goats. Food Chem. 2018; 254:326-332. https://doi. org/10.1016/j.foodchem.2018.02.041

34. Yang $Y$, Dong G, Wang $Z$, Wang J, Zhang $Z$, Liu J. Rumen and plasma metabolomics profiling by UHPLC-QTOF/MS revealed metabolic alterations associated with a highcorn diet in beef steers. PloS One. 2018; 13(11). https://doi.org/10.1371/journal. pone. 0208031

35. Giuffrida-Mendoza M, de Moreno L, HuertaLeidenz N, Uzcátegui-Bracho S, ValeroLeal K, Romero S, Rodas-González A. Cholesterol and fatty acid composition of longissimus thoracis from water buffalo (Bubalus bubalis) and Brahman-influenced cattle raised under savannah conditions. Meat Sci. 2015; 106:44-49. https://doi. org/10.1016/j.meatsci.2015.03.024 